\title{
Ranking Rate Control and Rhythm Control Therapies for Heart Failure Patients With Atrial Fibrillation: A Network Meta-Analysis of Randomized Controlled Trials
}

\author{
Cong Zhang, Hongxing Luo, Yu Xu* , Juntao Wang, Yanan Shi, Pengfei Zhang \\ Department of Cardiology, Zhengzhou University People's Hospital, Zhengzhou, Henan, China
}

*Corresponding Author: Yu Xu, Department of Cardiology, Zhengzhou University People's Hospital, Zhengzhou, Henan, China, Tel: +86 13653850619; E-mail: 2125089514@qq.com

Received: 08 March 2018; Accepted: 14 March 2018; Published: 18 March 2018

\begin{abstract}
Background: Rate control and rhythm control have demonstrated similar benefits for heart failure (HF) patients with atrial fibrillation (AF), but no study has attempted to rank their benefits. We aim to conduct a network metaanalysis of the efficacies of rate control and rhythm control therapies for HF patient with AF.
\end{abstract}

Methods: We searched PubMed, Embase, and Cochrane Library with various combinations of "rate control", "rhythm control", "atrial fibrillation" and "heart failure". The retrieved records were screened by "PatientIntervention-Control-Outcome" principle. The eligible randomized controlled trials were extracted for basic characteristics, outcomes data, and synthesized with random-effects model.

Results: Eleven trials involving 2,086 patients and 8 rate control and rhythm control therapies were included. The interventions for all-cause mortality reduction were ranked as follows: catheter ablation (mean rank [MR] 2.3, surface under the cumulative ranking curves [SUCRA] 81.1\%), atrioventricular node ablation and pacemaker (MR 3.1, SUCRA 70.0\%), $\beta$-blocker (MR 3.9, SUCRA 58.8\%), optimal medical treatment (MR 3.9, SUCRA 57.9\%), amiodarone (MR 4.3, SUCRA 52.9\%), placebo (MR 4.5, SUCRA 50.3\%), digoxin (MR 6.8, SUCRA 16.7\%), $\beta$ blocker and/or digoxin (MR 7.1, SUCRA 12.2\%).

Conclusion: In HF patients with AF, catheter ablation may be the most effective therapy for rate control and rhythm control, while digoxin is probably harmful.

Keywords: Atrial Fibrillation; Heart Failure; Rate Control; Rhythm Control; Network Meta-Analysis 


\section{Introduction}

Atrial fibrillation (AF) and heart failure (HF) are global cardiovascular epidemics with increasing incidence and socioeconomic burdens [1]. The prevalence of AF in HF patients is high: $53 \%, 60 \%$ and $65 \%$ in HF with preserved, midrange and reduced ejection fraction, respectively [2]. AF increases the all-cause mortality, rehospitalization, and cerebrovascular attacks in HF patients of any ejection fraction group [3]. Both diseases share similar pathophysiological changes (e.g, structural cardiac remodeling and activation of neurohormonal mechanisms) and risk factors (e.g, aging, hypertension and obesity) [4].

Rate control and rhythm control are two major goals of AF treatment. Heart rate can be controlled by medications such as $\beta$-blocker, calcium channel blockers and digoxin, and non-medication strategies such as atrioventricular node ablation and pacemaker (AVNP). Heart rhythm can be controlled by antiarrhythmic drugs (class I, class III and multichannel blockers), catheter ablation (CA) and direct current cardioversion (DCC). A previous trial of AF and congestive HF has demonstrated no significant differences between rate control ( $\beta$-blocker, digoxin, and AVNP; $\mathrm{n}=694$ ) and rhythm control (amiodarone, sotalol, dofetilide, DCC, and permanent pacemaker; $\mathrm{n}=682$ ) for all-cause mortality [5]. The meta-analysis about rate control vs. rhythm control ( $\beta$-blocker, calcium channel blockers and digoxin vs. antiarrhythmic agents and DCC) in AF and HF patients has shown no significant differences in mortality [6]. However, no study has been conducted to rank the efficacy of rate control and rhythm control therapies in mortality reduction.

This study aims to use network meta-analysis of randomized controlled trials (RCTs) to compare the efficacies of rate control and rhythm control therapies for patients with AF and HF.

\section{Methods}

\subsection{Database searches}

On July 31, 2017, we searched for records in PubMed, Embase, and Cochrane Library using the various combinations of "rate control", "rhythm control", "atrial fibrillation", and "heart failure" (Supplement 1). We restricted the publication language for English, but the publication date was not restricted.

\subsection{Study selection}

After removal of duplicates, article type was firstly screened to include the original articles. Secondly, we screened the remaining records by PICO (Patient-Intervention-Control-Outcome) principle, using the following criteria: 1) AF and HF patients; 2) rate control or rhythm control therapies; 3) the outcomes included all-cause mortality. Thirdly, the reference lists of the eligible articles were checked to find potentially relevant studies. Fourthly, we eliminated the non- randomized controlled trial and created a network evidence, including all rate control and rhythm control therapies from the remaining RCTs, then the RCTs with isolated therapies were removed because they could not connect with the others. 


\subsection{Data extraction}

For each record, baseline characteristics and outcome data were extracted (Supplements 2, 3). Some articles showed the data only available in the figures, and they were extracted by a proven reliable tool WebPlotDigitizer [7].

\subsection{Statistical analysis}

The dichotomous outcome variables were compared with odd ratios (OR) with $95 \%$ credible intervals (CrIs) by means of network meta-analysis with random-effects model. Effect sizes (ES) were calculated to compare the differences of the means of continuous variables between two groups. Tau presented the between-trial variance and was as a measure of the heterogeneity between trials in the network for outcome and comparison. A tau estimates of 0.04 may be interpreted as a low, 0.14 as a moderate and 0.40 as a high degree of heterogeneity between trials. The direct comparisons with each trial, the comparisons of all available treatments across trials, and the comparison of one intervention against the others were performed by the network meta-analysis with consistency model. Placebo was used as the common comparator in the network model. The contribution plot was used to present the influence of each direct comparison to the estimation of the overall network effects, of which weighted squares and the respective percentages represented the contribution of each direct comparison. Rank graphs, surface under the cumulative ranking curves (SUCRA) and mean ranks (MR) were estimated to rank the intervention hierarchy in the network meta-analysis. The comparison-adjusted funnel plot was used to analyze publication bias and small study effects.

For this study, the outcome was all-cause mortality. The several metrics for each intervention were calculated to estimate treatment effects. An intervention with a mean rank of 0 had the most favorable effect. Treatments in networks were also ranked using probabilities, with high probabilities representing favorable treatments. SUCRA is an indicator of the efficacy of a treatment. A value of 100 percent represented the most effective treatment, whereas 0 represented the least effective treatment. All statistical analyses were performed in Stata version 14.0.

\section{Results}

We primarily obtained 2193 records, including 930, 349, and 914 records from PubMed, Cochrane Library, and Embase databases, respectively. After removal of duplicates, 1175 records were screened based on article type and PICO strategies, and 16 studies were included. Then we checked the reference lists of the 16 studies and identified 5 relevant studies. Finally, we eliminated 5 non-RCTs and created the evidence network, including all rate and rhythm control therapies from the remaining $16 \mathrm{RCTs}$, so $5 \mathrm{RCTs}$ with isolated therapies were removed. Therefore, 11 RCTs investigating 8 different rate control or rhythm control therapies were included in this network meta-analysis (Figure 1) [8-18]. The 11 RCTs involving 2,084 patients were conducted during 2001-2016, of which 6 in UK, 3 in US, 1 in France, and 1 in Turkey. The mean age of participants was 66.2 years and the mean follow-up time was 14 months (Supplement 2). The quality of the included studies was assessed by the Cochrane Collaboration's risk of bias assessment tool (Supplement 4) [19]. 


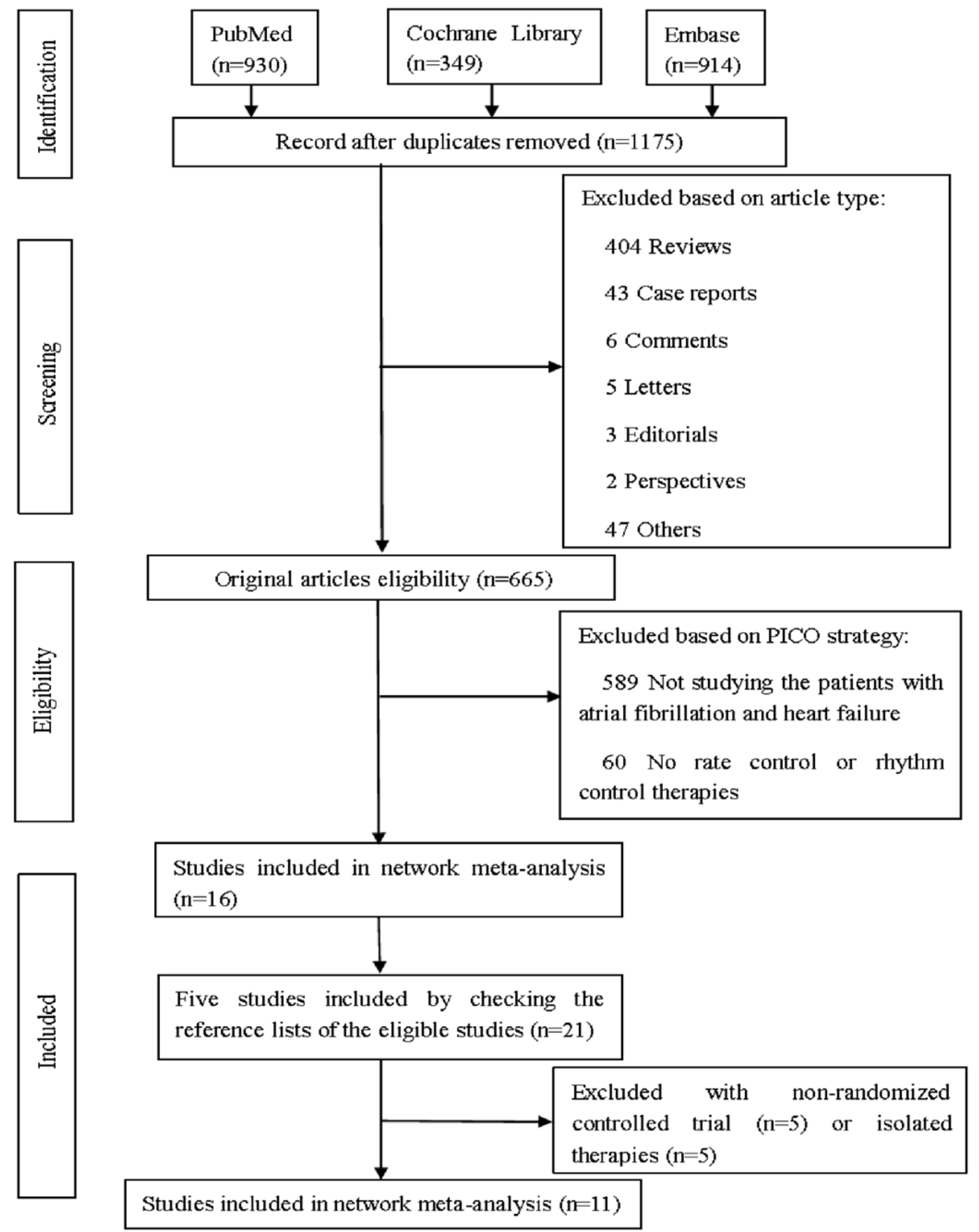

Figure 1: PRISMA flowchart of study selection

$\mathrm{PICO}=$ Patient-Intervention-Control-Outcome

Eight different interventions were collected from the 11 eligible RCTs: 1) placebo ( $\mathrm{n}=693), 2)$ amiodarone $(\mathrm{n}=211)$, 3) atrioventricular node ablation and pacemaker (AVNP, $n=40)$, 4) $\beta$-blocker $(n=733), 5) \beta$-blocker and/or digoxin $(\mathrm{BD}, \mathrm{n}=124), 6$ ) catheter ablation $(\mathrm{CA}, \mathrm{n}=217), 7)$ digoxin $(\mathrm{n}=23), 8)$ optimal medical treatment (OMT, $\mathrm{n}=43$ ). 
Eleven RCTs involving 2084 patients assessed all-cause mortality across 8 different interventions in this network meta-analysis. We drew a network map to show which treatments were directly compared against other treatments (Figure 2). The between-trial variance was evaluated with a tau estimate of 0 interpreted as a low degree of heterogeneity, and there was no significant inconsistency in this network meta-analysis $(\mathrm{P}=0.0847>0.05$, Supplement 5). A consistency model could be fitted to perform network meta-analysis in our study. The contribution plot showed the most informative direct evidence was CA vs amiodarone with an overall contribution of $20.9 \%$ to the network estimates (Supplement 6). The forest plots of direct and indirect comparison were presented in Figure 3. The rank graphs of all rate control and rhythm control therapies were shown in Figure 4. The comparisons of allcause mortality between all rate control and rhythm control therapies were shown in Supplement 7.

\section{All-cause mortality (11 RCTs, 2084 patients)}

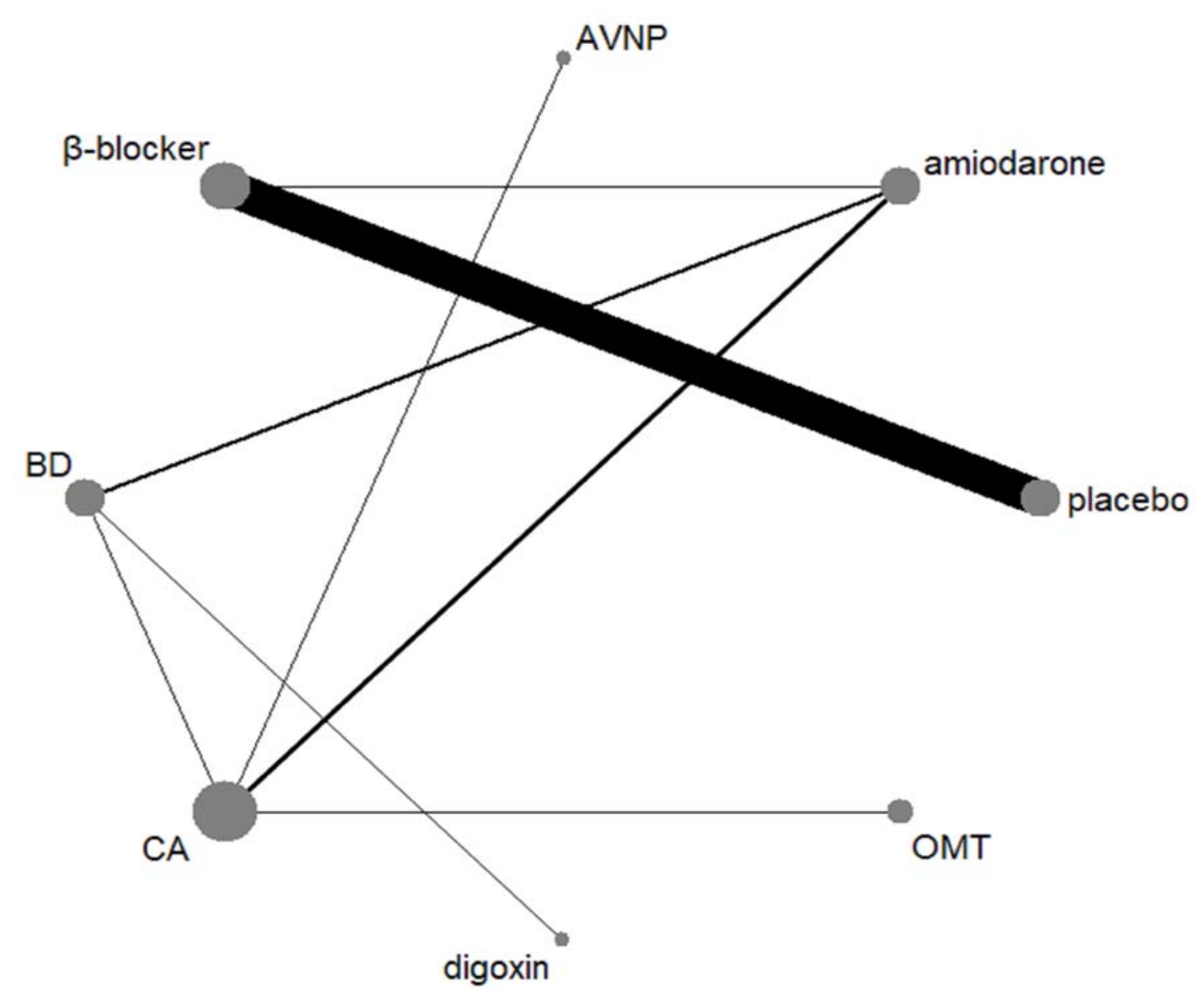

Figure 2: Network of eligible comparisons included in the analyses for outcomes

The size of every circle is proportional to the number of assigned patients and indicates the sample size. The lines link direct compared intervention, the width of which corresponds to the number of studies that assessed the comparison. $\mathrm{RCT}=$ randomized controlled trial. $\mathrm{AVNP}=$ atrioventricular node ablation and pacemaker. $\mathrm{BD}=\beta$-blocker and/or digoxin. $\mathrm{CA}=$ catheter ablation. $\mathrm{OMT}=$ optimal medical treatment. 


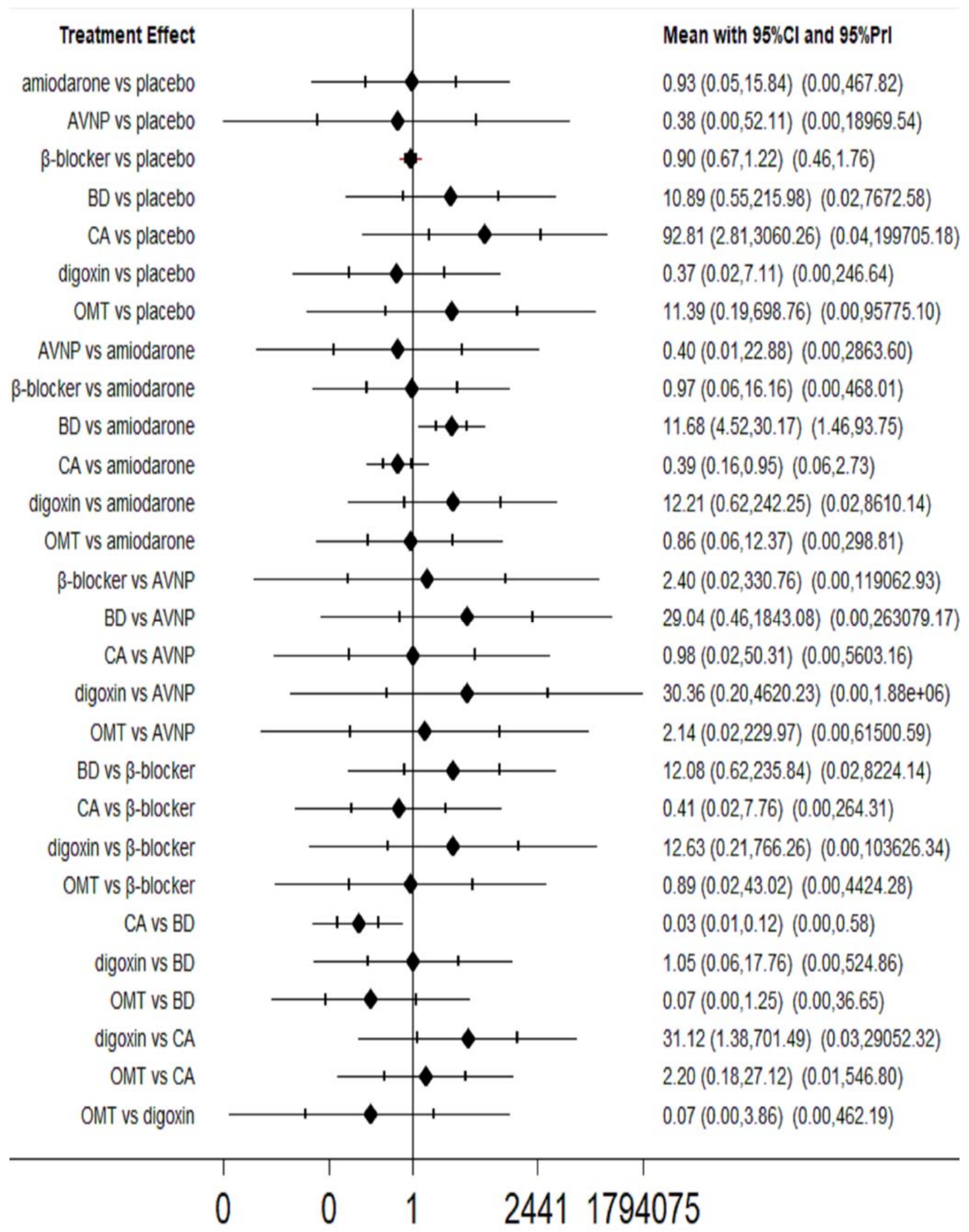

Figure 3: Estimates of the treatment effects on all-cause mortality of different rate control and rhythm control therapies.

$\mathrm{AVNP}=$ atrioventricular node ablation and pacemaker. $\mathrm{BD}=\beta$-blocker and/or digoxin. $\mathrm{CA}=$ catheter ablation. $\mathrm{OMT}=$ optimal medical treatment. 

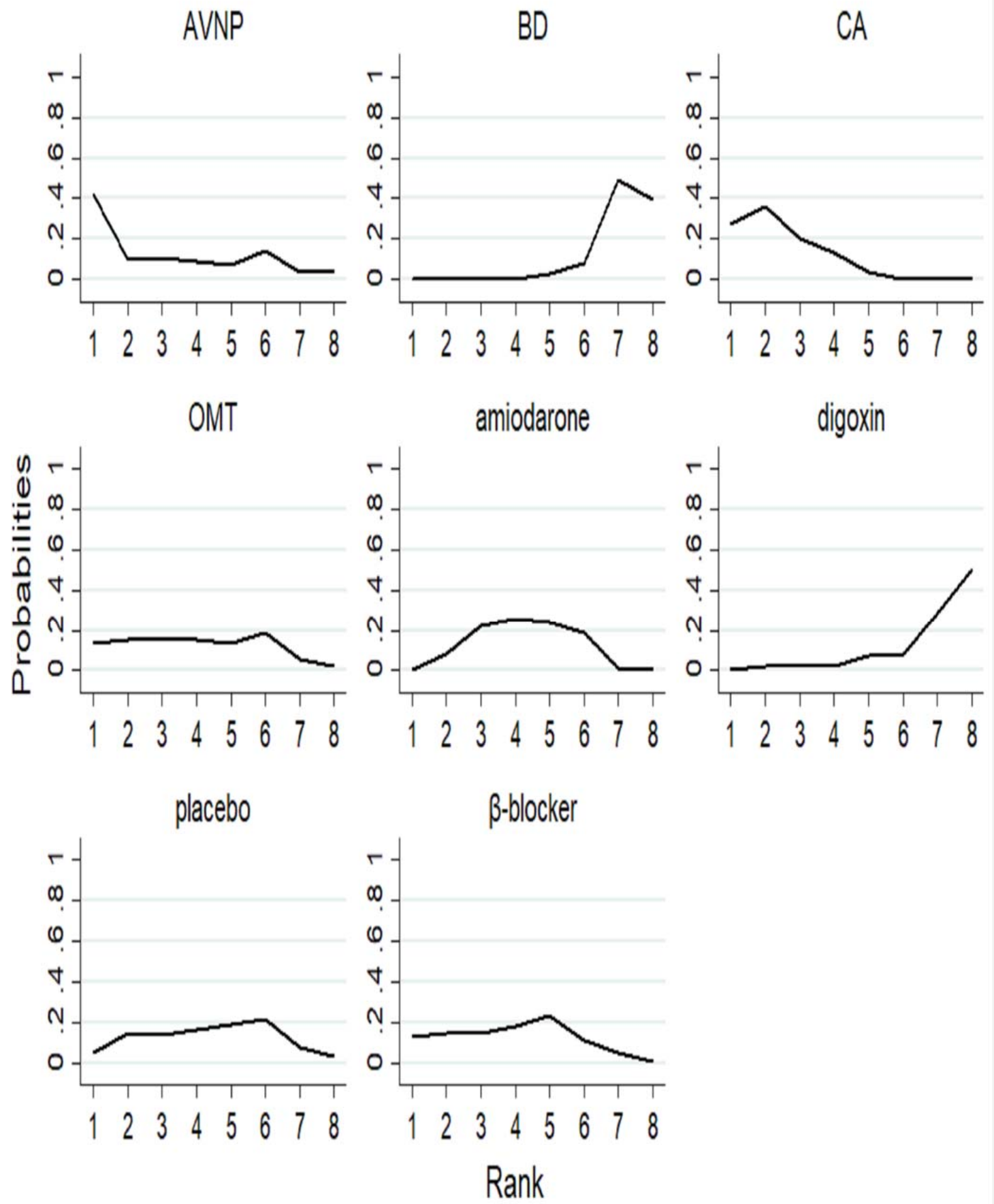

\section{Graphs by Treatment}

Figure 4: Cumulative ranking plot of the rate control and rhythm control therapies compared by all-cause mortality

$1=$ placebo, $2=$ amiodarone, $3=\mathrm{AVNP}, 4=\beta$-blocker, $5=\mathrm{BD}, 6=\mathrm{CA}, 7=$ digoxin, $8=\mathrm{OMT}$. AVNP=atrioventricular node ablation and pacemaker. $\mathrm{BD}=\beta$-blocker and/or digoxin. $\mathrm{CA}=$ catheter ablation. $\mathrm{OMT}=$ optimal medical treatment.

The rank of intervention for reducing all-cause mortality was as follows (Table 1): CA (MR 2.3, SUCRA 81.4\%); AVNP (MR 3.1, SUCRA 70.6\%); $\beta$-blocker (MR 3.8, SUCRA 59.8\%); OMT (MR 3.9, SUCRA 58.7\%); 
amiodarone (MR 4.3, SUCRA 53.4\%); placebo (MR 4.4, SUCRA 51.4\%); digoxin (MR 7.0, SUCRA 13.9\%); BD (MR 7.2, SUCRA 10.8\%). Comparison-adjusted funnel plots showed no significant evidence of publication bias or small-study effects (Supplement 8).

\begin{tabular}{|l|l|l|}
\hline & \multicolumn{2}{|l|}{ All-cause mortality } \\
\hline Treatment & SUCRA (\%) & MR \\
\hline placebo & 51.4 & 4.4 \\
\hline amiodarone & 53.4 & 4.3 \\
\hline AVNP & 70.6 & 3.1 \\
\hline$\beta$-blocker & 59.8 & 3.8 \\
\hline BD & 10.8 & 7.2 \\
\hline CA & 81.4 & 2.3 \\
\hline digoxin & 13.9 & 7.0 \\
\hline OMT & 58.7 & 3.9 \\
\hline
\end{tabular}

Table 1: Rank chart of efficacy outcomes

SUCRA $=$ the surface under the cumulative ranking line. $\mathrm{MR}=$ mean rank. $\mathrm{AVNP}=$ atrioventricular node ablation and pacemaker. $\mathrm{BD}=\beta$-blocker and/or digoxin. $\mathrm{CA}=$ catheter ablation. $\mathrm{OMT}=$ optimal medical treatment.

\section{Discussion}

To the best knowledge, this is the first study ranking the efficacies of rate control and rhythm control therapies in HF patients with AF. Our principal findings are: 1) CA, AVNP, $\beta$-blocker, OMT, and amiodarone were associated with significantly lower all-cause mortality than placebo; 2) CA may be the most effective therapy for reducing all-cause mortality; 3) digoxin may not be effective for reducing all-cause mortality.

Rate control and rhythm control are often required for HF patients with AF. Rate control is determined by the conduction of atrioventricular node and the balance of sympathetic and parasympathetic activities. Rate control drugs are divided into three groups: $\beta$-blocker (blocking sympathetic activities of the atrioventricular node), nondihydropyridine calcium channel blocker (blocking calcium channels to increase the refractory period of atrioventricular node), and cardiac glycoside (increasing parasympathetic activity and slowing atrioventricular node conduction). They may be used alone or in combination. AVNP can effectively control ventricular rate when conventional therapies fail or serious drug-related adverse effects occur. Rhythm control aims to restore and maintain sinus rhythm and includes DCC, class I antiarrhythmic drugs, class III antiarrhythmic drugs, and multichannel blockers [4]. CA is used for refractory symptomatic AF patients who are irresponsive to at least one class I or class III antiarrhythmic drugs and who are intolerant to other therapies [4].

Rhythm control therapies are effective when sinus rhythm is restored and maintained for at least 1 year [13]. Recurrence rate at 1 year recorded in the published studies is $17 \%$ in the Atrial Fibrillation Follow-up Investigation 
of Rhythm Management study, $43 \%$ in the Strategies of Treatment of Atrial Fibrillation study and $44 \%$ in the Pharmacological Intervention in Atrial Fibrillation study, respectively [20-22]. Moreover, HF is usually accompanied by dilated atria and structural heart diseases which give rise to a high recurrence rate [23]. Low dosage $(\leq 200 \mathrm{mg} / \mathrm{d})$ is more effective and safer than sotalol or propafenone for maintaining sinus rhythm for 1 year [24]. The adverse effects of amiodarone are related to the dosage and duration of therapy. The dosage of amiodarone to maintain sinus rhythm should be less than $200 \mathrm{mg} / \mathrm{d}$ in the included RCTs [11, 13, 18]. However, amiodarone may not be safe enough for HF patients with AF in long term [25]. Pulmonary toxicity, hepatic toxicity, thyroid toxicity, and severe arrhythmia have been reported in the long-term use of amiodarone. The follow-up time of most patients (181/211) treated with amiodarone was more than 1 year in our study [11, 18]. Beta-blocker has been shown to be ineffective in reducing the all-cause mortality of HF patients with AF in a recent meta-analysis [26]. The main site of $\beta$-blocker to reduce heart rate in patients $\mathrm{AF}$ is atrioventricular node, but sinus node is the main target for heart failure patients with sinus rhythm. Therefore, $\beta$-blocker is less effective in those with sinus rhythm than AF. To achieve the same degree of rate control, the dosage of $\beta$-blocker may be higher in patients with AF than those with sinus rhythm. However, the higher dosage of $\beta$-blocker also weakens the myocardial contractility and reduces cardiac output. Digoxin significantly increased all-cause mortality in AF patients with HF as shown by a recent meta-analysis [27]. The therapeutic window of digoxin is narrow. However, only one trial [10] monitored the serum digoxin concentration (SDC) in our 4 included trials of digoxin. The mean SDC was more than $1.2 \mathrm{ng} / \mathrm{ml}$ both in the baseline and endpoint. The SDC $0.5-0.9 \mathrm{ng} / \mathrm{ml}$ is associated with all-cause mortality reduction, but digoxin increases all-cause mortality when SDC is $>1.2 \mathrm{ng} / \mathrm{ml}$ in HF patients [28]. A high SDC relates to cardiotoxicity, and leads to life-threatening tachyarrhythmias or bradyarrhythmias. The unusual activation of platelets and endothelial cells may also lead to unfavorable effects in the patients taking digoxin [29].

$\mathrm{CA}$ and AVNP are interventional therapies with potential interventional complications and direct injuries to heart structures. The complication rate of CA for AF patients in a large prospective study was $5.2 \%$ [30], which is $9.2 \%$ in this study $[12,14,16-18]$. The complication rate of AVNP for the AF patients with HF in our study was $17.5 \%$ [12]. AVNP is safe in AF patients but its effects in HF patients with AF remain unknown [31]. The salutary effect of AVNP on left ventricular systolic function, diastolic function, and cardiac output is significantly related to the strict control of heart rate without negative inotropic effects [32]. It also enhances diastolic filling time and reverses tachycardia-induced cardiomyopathy [33]. The previous meta-analysis has suggested that CA improved cardiac function and quality of life in HF patients with AF as compared with rate control therapy [34]. CA provides the opportunity to achieve and maintain sinus rhythm with a relatively low risk of complications and side effects. The continually increasing toxicity risks with amiodarone should be seriously considered for patients requiring long-term treatments. CA may be an alternative to amiodarone. There are no evidence comparing CA and rate control drugs for all-cause mortality reduction for HF patients with AF. As a result, mortality data may be insufficient. In the future, the studies about the mortality of HF patients with $\mathrm{AF}$ who receive different rate control and rhythm control therapies are needed.

Firstly, we have included a relatively small number of studies about rate control and rhythm control in HF patients with AF. Secondly, although we have referenced numerous documents to optimize our study protocol and search 
strategies, our meta-analysis may not have covered all studies related to rate control and rhythm control. Thirdly, as a network meta-analysis, our results have been inevitably influenced by the quality of the included studies.

\section{Conclusion}

In HF patients with AF, catheter ablation may be the most effective therapy for rate control and rhythm control, while digoxin is probably harmful. Our study results should be tested by more clinical studies in the future.

\section{Acknowledgement}

None.

\section{Conflicts of interest}

None.

\section{Supplementary File}

Download the supplementary file here

http://www.cardiolcardiovascmed.com/10.26502/fccm.92920033.pdf

\section{References}

1. Schnabel RB, et al. 50 year trends in atrial fibrillation prevalence, incidence, risk factors, and mortality in the Framingham Heart Study: a cohort study. Lancet 386 (2015): 154-162.

2. Lam C, BT Santema and AA Voors. Atrial Fibrillation in Heart Failure: A Common and Deadly Combination. JACC Heart Fail 5 (2017): 575-577.

3. Sartipy U, et al. Atrial Fibrillation in Heart Failure With Preserved, Mid-Range, and Reduced Ejection Fraction. JACC Heart Fail 5 (2017): 565-574.

4. Kirchhof $\mathrm{P}$, et al. ESC Guidelines for the management of atrial fibrillation developed in collaboration with EACTS. Europace 18 (2016): 1609-1678.

5. Roy D, et al. Rhythm Control versus Rate Control for Atrial Fibrillation and Heart Failure. New England Journal of Medicine 358 (2008): 2667-2677.

6. Caldeira D, C David and C Sampaio. Rate vs rhythm control in patients with atrial fibrillation and heart failure: a systematic review and meta-analysis of randomised controlled trials. Eur J Intern Med 22 (2011): 448-455.

7. Drevon D, SR Fursa and AL Malcolm. Intercoder Reliability and Validity of WebPlotDigitizer in Extracting Graphed Data. Behav Modif 41 (2017): 323-339.

8. Lechat $\mathrm{P}$, et al. Heart rate and cardiac rhythm relationships with bisoprolol benefit in chronic heart failure in CIBIS II Trial. Circulation 103 (2001): 1428-1433.

9. Joglar JA, et al. Effect of carvedilol on survival and hemodynamics in patients with atrial fibrillation and left ventricular dysfunction: retrospective analysis of the US Carvedilol Heart Failure Trials Program. Am Heart J 142 (2001): 498-501. 
10. Khand AU, et al. Carvedilol alone or in combination with digoxin for the management of atrial fibrillation in patients with heart failure? J Am Coll Cardiol 42 (2003): 1944-1951.

11. Okcun B, et al. Comparison of rate and rhythm control in patients with atrial fibrillation and nonischemic heart failure. Jpn Heart J 45 (2004): 591-601.

12. Khan M.N, et al. Pulmonary-vein isolation for atrial fibrillation in patients with heart failure. N Engl J Med 359 (2008): 1778-1785.

13. Shelton RJ, et al. A randomised, controlled study of rate versus rhythm control in patients with chronic atrial fibrillation and heart failure: (CAFE-II Study). Heart 95 (2009): 924-930.

14. MacDonald MR, et al. Radiofrequency ablation for persistent atrial fibrillation in patients with advanced heart failure and severe left ventricular systolic dysfunction: a randomised controlled trial. Heart 97 (2011): 740747.

15. Mulder BA, et al. Effect of nebivolol on outcome in elderly patients with heart failure and atrial fibrillation: insights from SENIORS. Eur J Heart Fail 14 (2012): 1171-1178.

16. Jones DG, et al. A randomized trial to assess catheter ablation versus rate control in the management of persistent atrial fibrillation in heart failure. J Am Coll Cardiol 61 (2013): 1894-1903.

17. Hunter RJ, et al. A randomized controlled trial of catheter ablation versus medical treatment of atrial fibrillation in heart failure (the CAMTAF trial). Circ Arrhythm Electrophysiol 7 (2014): 31-38.

18. Di Biase L, et al. Ablation Versus Amiodarone for Treatment of Persistent Atrial Fibrillation in Patients With Congestive Heart Failure and an Implanted Device: Results From the AATAC Multicenter Randomized Trial. Circulation 133 (2016): 1637-1644.

19. Higgins JP, et al. The Cochrane Collaboration's tool for assessing risk of bias in randomised trials. BMJ 343 (2011): d5928.

20. Wyse DG, et al. A comparison of rate control and rhythm control in patients with atrial fibrillation. N Engl J Med 347 (2002): 1825-1833.

21. Hohnloser $\mathrm{SH}, \mathrm{KH}$ Kuck and J Lilienthal. Rhythm or rate control in atrial fibrillation--Pharmacological Intervention in Atrial Fibrillation (PIAF): a randomised trial. Lancet 356 (2000): 1789-1794.

22. Carlsson J, et al. Randomized trial of rate-control versus rhythm-control in persistent atrial fibrillation: the Strategies of Treatment of Atrial Fibrillation (STAF) study. J Am Coll Cardiol 41 (2003): 1690-1696.

23. Dittrich, H.C, et al, Echocardiographic and clinical predictors for outcome of elective cardioversion of atrial fibrillation. Am J Cardiol 63 (1989): 193-197.

24. Roy D, et al. Amiodarone to prevent recurrence of atrial fibrillation. Canadian Trial of Atrial Fibrillation Investigators. N Engl J Med 342 (2000): 913-920.

25. Torp-Pedersen C, et al. The safety of amiodarone in patients with heart failure. J Card Fail 13 (2007): 340345.

26. Kotecha D, et al. Efficacy of beta blockers in patients with heart failure plus atrial fibrillation: an individualpatient data meta-analysis. Lancet 384 (2014): 2235-2243.

27. Qureshi W, et al. Systematic review and meta-analysis of mortality and digoxin use in atrial fibrillation. Cardiol J 23 (2016): 333-343. 
28. Ahmed A, et al. Digoxin and reduction in mortality and hospitalization in heart failure: a comprehensive post hoc analysis of the DIG trial. Eur Heart J 27 (2006): 178-186.

29. Chirinos JA, et al. Digoxin use is associated with increased platelet and endothelial cell activation in patients with nonvalvular atrial fibrillation. Heart Rhythm 2 (2005): 525-529.

30. Bohnen $\mathrm{M}$, et al. Incidence and predictors of major complications from contemporary catheter ablation to treat cardiac arrhythmias. Heart Rhythm 8 (2011): 1661-1666.

31. Wood MA, et al. Clinical outcomes after ablation and pacing therapy for atrial fibrillation : a meta-analysis. Circulation 101 (2000): 1138-1144.

32. Daoud EG, et al. Effect of an irregular ventricular rhythm on cardiac output. Am J Cardiol 78 (1996): 14331436.

33. Shinbane JS, et al. Tachycardia-induced cardiomyopathy: a review of animal models and clinical studies. J Am Coll Cardiol 29 (1997): 709-715.

34. Al HS, et al. Catheter Ablation for Atrial Fibrillation in Heart Failure Patients: A Meta-Analysis of Randomized Controlled Trials. JACC Clin Electrophysiol 1 (2015): 200-209.

Citation: Cong Zhang, Hongxing Luo, Yu Xu, Juntao Wang, Yanan Shi, Pengfei Zhang. Ranking Rate Control and Rhythm Control Therapies for Heart Failure Patients With Atrial Fibrillation: A Network MetaAnalysis of Randomized Controlled Trials. Cardiology and Cardiovascular Medicine 2 (2018): 027-038.

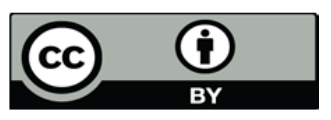

This article is an open access article distributed under the terms and conditions of the Creative Commons Attribution (CC-BY) license 4.0 